\title{
Global Existence of Smooth Solutions and Stability of Solitary Waves for a Generalized Boussinesq Equation
}

\author{
Jerry L. Bona ${ }^{1,2 \star}$ and Robert L. Sachs ${ }^{1}$
}

1 Department of Mathematics, The Pennsylvania State University, University Park, PA 16802, USA

2 Applied Research Laboratory, The Pennsylvania State University, University Park, PA 16802, USA

\begin{abstract}
Certain generalizations of one of the classical Boussinesq-type equations,

$$
u_{t t}=u_{x x}-\left(u^{2}+u_{x x}\right)_{x x}
$$

are considered. It is shown that the initial-value problem for this type of equation is always locally well posed. It is also determined that the special, solitary-wave solutions of these equations are nonlinearly stable for a range of their phase speeds. These two facts lead to the conclusion that initial data lying relatively close to a stable solitary wave evolves into a global solution of these equations. This contrasts with the results of blow-up obtained recently by Kalantarov and Ladyzhenskaya for the same type of equation, and casts additional light upon the results for the original version $(*)$ of this class of equations obtained via inverse-scattering theory by Deift, Tomei and Trubowitz.
\end{abstract}

\section{Introduction}

In the 1870 's, Boussinesq derived some model equations for the propagation of small amplitude, long waves on the surface of water. These equations possess special, travelling-wave solutions called solitary waves, and Boussinesq's theory was the first to give a satisfactory, scientific explanation of the phenomenon of solitary waves discovered by Scott-Russell and reported more than thirty years earlier. In one of his papers (Boussinesq, 1872) he also proposed what we would now call a Lyapunov function, which he argued was connected to the stability of these solitary waves.

The original equation due to Boussinesq is not the only mathematical model for small-amplitude, planar, long waves on the surface of water. Different choices of the dependent variables, plus the possibility of modifying lower order terms by the use of the leading order relationships can lead to a whole range of equations,

\footnotetext{
* Work partially supported by the National Science Foundation
} 
all of which have the same formal validity (cf. Bona and Smith, 1976, for a discussion of various alternatives). All of these models possess one obvious characteristic, however, which is that they are perturbations of the linear wave equation that take account of small effects of nonlinearity and dispersion.

Despite that status of Boussinesq-type equations as the first model for nonlinear, dispersive wave propagation, the mathematical theory for such equations is not nearly so complete as is the case for the Korteweg-de Vries-type equations. The latter equations are similar in scope to the Boussinesq equations, but they are first order in the temporal variable instead of second order.

Part of the reason for the relative paucity of results concerning Boussinesq-type equations may be due to the recently discovered fact that the initial-value problem is not always globally well posed. There exist initial wave and velocity profiles which are themselves smooth, but for which the solution that emanates from them loses regularity in finite time, becoming infinite in fact (see Kalantarov and Ladyzhenskaya, 1978, and Deift et al. 1982).

In the present article, a generalization of one of the Boussinesq-type equations is considered which arises in the modelling of nonlinear strings, namely

$$
u_{t t}-u_{x x}+\left(f(u)+u_{x x}\right)_{x x}=0
$$

With regard to this class of equations, the following program will be pursued. First, a theory of local existence of smooth solutions for the initial-value problem for (1.1a) is briefly recounted in which the auxiliary conditions

$$
u(x, 0)=u_{0}(x) \text { and } u_{t}(x, 0)=u_{1}(x)
$$

are specified. Next, properties are presented which allow the inference that local smooth solutions may be extended uniquely to solutions defined globally in time. Then, the nonlinear stability of the solitary-wave solutions of these equations is proved for a certain range of the wave's speed of propagation and pure power nonlinearities. Finally, local existence coupled with the stability result is shown to imply the conditions that lead to global existence, at least for initial data that resembles one of the stable solitary waves.

The plan of the paper is as follows. Section 2 contains an abbreviated description of the local existence theory for these equations along with the theorem specifying sufficient conditions for a local solution to be globally continuable. The stability theory is presented in Sect. 3, following the lines laid down by Grillakis et al. (1987). These results are combined in Sect. 4 to deduce the aforementioned global well posedness. A few concluding remarks are to be found in the last Section.

\section{Local Existence Theory}

The present section features two accomplishments. A theorem asserting the local well-posedness of the initial-value problem (1.1) is stated and sufficient conditions are established that ensure the local solution to be continuable to a smooth solution defined globally in time. The well-posedness theorem is a straightforward consequence of the abstract techniques of Kato $(1975,1983)$ for quasi-linear evolution equations, and consequently the proof is omitted. The theorem concerned with 
global existence uses the local theory, a regularizing operator, and energy-type estimates.

To apply Kato's theory to the initial-value problem (1.1), view the equation in an equivalent form as a system of equations, namely

$$
\left.\begin{array}{c}
u_{t}=v_{x} \\
v_{t}=\left(u-u_{x x}-f(u)\right)_{x}
\end{array}\right\} \text { for } x \in \mathbb{R} . \quad t>0
$$

with

$$
\left.\begin{array}{l}
u(x, 0)=u_{0}(x) \\
v(x, 0)=v_{0}(x)
\end{array}\right\} \text { for } x \in \mathbb{R}
$$

where $f: \mathbb{R} \rightarrow \mathbb{R}$ is a $C^{\infty}$-function with $f(0)=0$. (The latter normalization is made without loss of generality since $f(u)$ only appears differentiated.) The particular form of the nonlinearity is not important for the local theory, though in the next sections substantial restrictions on $f$ will be imposed.

The notation employed is standard, and may be found explained in detail in Lions and Magenes (1968). Except for the abbreviation $\|g\|_{s}$ for the $H^{s}(\mathbb{R})$-norm of a function $g$, the usual norm on some Banach space $X$ of functions will be denoted $\|g\|_{X}$.

In discussing the local existence theory it is convenient to introduce another abbreviation, namely, for $T>0$ a finite real number and $s$ an arbitrary Sobolev index, set

$$
X_{s}(T)=C\left(0, T ; H^{s+2}(\mathbb{R})\right) \cap C^{1}\left(0, T ; H^{s}(\mathbb{R})\right) .
$$

Theorem 1. Let $u_{0} \in H^{s+2}(\mathbb{R})$ and $v_{0} \in H^{s+1}(\mathbb{R})$ for some $s>1 / 2$. Denote by $Y$ the product space $H^{s+2}(\mathbb{R}) \times H^{s+1}(\mathbb{R})$. Then there exists $T>0$ which depends only upon $\left\|\left(u_{0}, v_{0}\right)\right\|_{Y}$, and unique functions $u \in X_{s}(T)$ and $v \in X_{s-1}(T)$ which solve the initial-value problem (2.1). Moreover, the pair $(u, v)$ depends continuously on $\left(u_{0}, v_{0}\right)$ in the sense that the mapping sending $\left(u_{0}, v_{0}\right)$ to the associated solution of $(2.1)$ is continuous from $Y$ into the space $X_{s}(T) \times X_{s-1}(T)$.

This theorem follows directly from the general results of Kato $(1975,1983)$ on quasi-linear evolution equations. The functional-analytic setting for Kato's theory consists of a pair of reflexive Banach spaces $X$ and $Y$, with $Y$ continuously and densely imbedded in $X$. A central role in the theory is played by a Banach-space isomorphism $S$ of $Y$ onto $X$, and the norms on these two spaces are chosen in such a way that $S$ is an isometry. The theory applies to the abstract, quasi-linear evolution equation

for $0<t$, with

$$
U_{t}+A(t, U) U=F(t, U)
$$

$$
U(0)=\varphi,
$$

where $\varphi$ is a given, initial value. The theory asserts that there exists a positive time $T$ such that (2.3) possesses a unique solution in $C(0, T ; Y) \cap C^{1}(0, T ; X)$, provided that certain assumptions are in force.

To apply Kato's theory to the situation envisaged in Theorem 1, take $X=H^{s}(\mathbb{R}) \times H^{s-1}(\mathbb{R})$ with $s>\frac{1}{2}$, take $Y$ as in the statement of the theorem, let 
$S=\left(I-\partial_{x}^{2}, I-\partial_{x}^{2}\right)$ with $I$ connoting the identity operator, let $A$ be the matrix of differential operators

$$
A=\left(\begin{array}{cc}
0 & -\partial_{x} \\
-\partial_{x}+\partial_{x}^{3} & 0
\end{array}\right),
$$

and take the nonlinear operator $F$ to be

$$
F=F(t, u, v)=\left(\begin{array}{c}
0 \\
-f(u)_{x}
\end{array}\right) .
$$

With this choice of $A$ and $F$, and writing

$$
U=\left(\begin{array}{l}
u \\
v
\end{array}\right),
$$

(2.3) reduces to (2.1) if $\varphi=\left(u_{0}, v_{0}\right)$, and it is straightforward to verify that the hypotheses appertaining to Kato's theory are valid.

The consequences of Theorem 1 need interpretation as regards the initial-value problem (1.1) for the second-order equation. An interesting point arises in this interpretation. To focus the discussion, a direct consequence of Theorem 1 is stated in the following corollary.

In the statement of the corollary, the following spaces come naturally to the fore. Let $T$ be positive and $s$ a Sobolev index as before. Define

$$
Y_{s}(T)=X_{s}(T) \cap C^{2}\left(0, T ; H^{s-2}(\mathbb{R})\right) .
$$

Corollary 2. Let $u_{0} \in H^{s+2}(\mathbb{R})$ and $v_{0} \in H^{s+1}(\mathbb{R})$, where $s>\frac{1}{2}$. Then there exists a $T>0$, depending only upon the norm of $\left(u_{0}, v_{0}\right) \in H^{s+2}(\mathbb{R}) \times H^{s+1}(\mathbb{R})$, and a unique function $u \in Y_{s}(T)$ which is a solution of Eq. (1.1a) in the distributional sense on $\mathbb{R} \times[0, T]$, and for which $u(\cdot, 0)=u_{0}$ and $u_{t}(\cdot, 0)=v_{0}^{\prime}$. The solution $u$ depends continuously upon the data $\left(u_{0}, v_{0}\right)$ in the sense that the mapping that associates to $\left(u_{0}, v_{0}\right)$ the solution $u$ is continuous from $H^{s+2}(\mathbb{R}) \times H^{s+1}(\mathbb{R})$ into $Y_{s}(T)$. If $s>\frac{5}{2}$, then the solution is classical, which means that all the derivatives featured in the equation exist pointwise and are jointly continuous functions of $x$ and $t$.

The restriction that the initial velocity $u_{t}(\cdot, 0)$ be equal to $v_{0}^{\prime}$ is mathematically somewhat unnatural. A more natural presumption is that both $u_{0}$ and $u_{1}$ in $(1.1 \mathrm{~b})$ be restricted simply as regards smoothness and decay to zero at infinity. A result similar to that given in Corollary 2 holds good in this case also, as the following theorem makes clear.

Theorem 3. Let $u_{0} \in H^{s+2}(\mathbb{R})$ and $u_{1} \in H^{s}(\mathbb{R})$, where $s>\frac{1}{2}$. Then there exists $T>0$, depending only upon the norm of $\left(u_{0}, u_{1}\right) \in H^{s+2}(\mathbb{R}) \times H^{s}(\mathbb{R})$, and a unique function $u \in Y_{s}(T)$ which is a solution of Eq. (1.1a) in the distributional sense on $\mathbb{R} \times[0, T]$, and for which $u(\cdot, 0)=u_{0}$ and $u_{t}(\cdot, 0)=u_{1}$. Moreover, the solution $u$ depends continuously upon $\left(u_{0}, u_{1}\right)$ in the sense that the mapping sending $\left(u_{0}, u_{1}\right)$ to $u$ is continuous from $H^{s+2}(\mathbb{R}) \times H^{s}(\mathbb{R})$ into $Y_{s}(T)$. If $s>\frac{5}{2}$, then the solution is classical in that all the derivatives featured in the equation exist pointwise and are jointly continuous functions of $x$ and $t$. 
This result may also be proved using Kato's theory, but the details are slightly different. Write (1.1) as a system in the form (2.3) with

$$
A(U)=\left(\begin{array}{cr}
0 & -1 \\
\left(1-\partial_{x}^{2}\right)^{2} & 0
\end{array}\right),
$$

and

$$
F(U)=\left(\begin{array}{c}
0 \\
\left(u-u_{x x}-f(u)\right)_{x x}
\end{array}\right),
$$

where $U$ is as in $(2.4 \mathrm{c})$ and the initial data $\varphi=\left(u_{0}, u_{1}\right)$. The system $(2.9)$ is formally equivalent to the initial-value problem (1.1), with no implied restriction on the initial data.

Take $X=H^{s}(\mathbb{R}) \times H^{s-2}(\mathbb{R}), Y=H^{s+2}(\mathbb{R}) \times H^{s}(\mathbb{R})$, and choose $S$ as before. With these choices, the hypotheses of Kato's theory are valid, and so the stated conclusions hold.

Remarks. Of course, Theorem 3 implies Corollary 2, but Theorem 1 and Theorem 3 appear to be separate results, neither of which implies the other.

The apparently trivial difference in the assumptions on the initial velocity are not without consequence as regards both the stability theory and the global existence theory. The stability theory relies upon the Hamiltonian form of the system (2.1). Indeed, the Hamiltonian functional

$$
H(u, v)=\frac{1}{2} \int_{-\infty}^{\infty}\left[v^{2}(x, t)+u^{2}(x, t)+u_{x}^{2}(x, t)-2 F(u(x, t))\right] d x
$$

where $F^{\prime}=f$ and $F(0)=0$, plays a central role in the analysis underlying our proof of stability. The stability theory, in turn, is crucial for the global existence theory. It is clear that $H$ will not be finite at the initial instant of time unless the initial data $u_{1}$ is the derivative of a square-integrable function. As the invariance of $H$ under the flow generated by the equation is used in an important way in our proof, we have thus far not been able to write a stability and global existence theory based upon the unrestricted problem (1.1) or its equivalent (2.9).

It is worth emphasis that all of these results imply the existence of solutions of the respective equation or system of equations that are only local in time. Note especially that in Theorem 1 and Corollary 2, the time of existence $T$ depends only upon $\left\|u_{0}\right\|_{s+2}+\left\|v_{0}\right\|_{s+1}$, whereas in Theorem 3 the existence time depends upon $\left\|u_{0}\right\|_{s+2}+\left\|u_{1}\right\|_{s}$. If a solution emanating from some particular initial data can be inferred to remain bounded in the appropriate norm, at least over bounded time intervals, it then follows by repetition of the local existence theorem that this solution extends to a global solution of the relevant initial-value problem. By the term "global solution" we mean that if $T^{*}$ denotes the maximum temporal interval of existence of the solution in question, so that for $T<T^{*}$, the solution lies in the function class set forth in the relevant theorem on the interval $[0, T]$, then $T^{*}=+\infty$.

A question of central interest to the qualitative analysis of an initial-value problem is whether or not solutions are necessarily global. In the case of the problem (1.1), the results of Kalantarov and Ladyzhenskaya (1978) show conclusive- 
ly that one cannot expect global solutions just on the basis of smoothness and decay assumptions. Our next result presents relatively weak a priori conditions that imply the local solutions obtained in either Theorem 1 or Theorem 3 to be global.

Theorem 4. Let $(u, v)$ be a local solution of either of the initial-value problems (2.1) or (2.9) corresponding to initial data $\left(u_{0}, v_{0}\right)$ as specified in Theorem 1 or Theorem 3, respectively. Suppose that there exists a constant $M_{0}$ such that $\|u(\cdot, t)\|_{1}+\|v(\cdot, t)\|_{0} \leqq M_{0}$ for all $t \in\left[0, T^{*}\right)$, where $\left[0, T^{*}\right)$ is the maximal temporal interval of existence of the solution $(u, v)$. Then $T^{*}=+\infty$, and the solution is global.

Proof. This result is proved in the context of Theorem 1. The proof in the context of Theorem 3 is very similar. According to the local existence theory as expounded in Theorem 1, the stated result will follow as soon as it is demonstrated that the norm of $(u, v)$ in $H^{s+2}(\mathbb{R}) \times H^{s+1}(\mathbb{R})$ remains bounded on the time interval $\left[0, T^{*}\right)$. To prove this, let $S_{\varepsilon}$ denote a smoothing operator, say $S_{\varepsilon}=\left(I-\varepsilon \partial_{x}^{2}\right)^{-1}$ for $\varepsilon>0$, and define $U=S_{\varepsilon} u, V=S_{\varepsilon} v$. Then the pair $(U, V)$ satisfies the system

with

$$
\left.\begin{array}{c}
U_{t}=V_{x} \\
V_{t}=U_{x}-U_{x x x}-G(U)_{x}
\end{array}\right\}
$$

$$
\left.\begin{array}{l}
U(x, 0)=S_{\varepsilon} u_{0}(x) \\
V(x, 0)=S_{\varepsilon} v_{0}(x)
\end{array}\right\},
$$

where $G(U)=S_{\varepsilon} f\left(S_{\varepsilon}^{-1} U\right)$. For fixed, positive $\varepsilon$ it follows that $(U, V) \in$ $X_{s+2}(T) \times X_{s+1}(T)$ for all $T<T^{*}$. Thus for any $r \leqq s+1$, the formal calculations below are justified (indeed, this is why $S_{\varepsilon}$ is applied at all).

Define $D^{r}$ for any $r \geqq 0$ as the Fourier-multiplication operator whose action on a function $f$ is given by

$$
\widehat{D^{r} f}(\xi)=|\xi|^{r} \widehat{f}(\xi) \text {. }
$$

Then $D^{r}$ is a self-adjoint, linear operator which is a bounded map of $H^{s}(\mathbb{R})$ to $H^{s-r}(\mathbb{R})$ for any real $s$.

Apply $\hat{o}_{x} D^{r}$ to the first equation in (2.11a) and multiply by $D^{r} U_{x}$. After integration with respect to $x$, this yields the relation

$$
\frac{1}{2} \frac{d}{d t}\left\|D^{r} U_{x}\right\|_{0}^{2}=\left(D^{r} U_{x}, D^{r} V_{x x}\right)
$$

Similarly, apply $D^{r}$ to the second equation in (2.11a), multiply by $D^{r} V$, and integrate with respect to $x$. Integration by parts, along with the fact that $D^{2}=-\partial_{x}^{2}$, leads to the equation

$$
\frac{1}{2} \frac{d}{d t}\left\|D^{r} V\right\|_{0}^{2}=\left(D^{r} V, D^{r} U_{x}\right)-\left(D^{r} U_{x}, D^{r} V_{x x}\right)-\left(D^{r} V, D^{r} \partial_{x} G(U)\right) .
$$

Adding (2.13) and (2.14), we come to the relation

$$
\begin{aligned}
\frac{1}{2} \frac{d}{d t}\left(\left\|D^{r} U_{x}\right\|_{0}^{2}+\left\|D^{r} V\right\|_{0}^{2}\right) & =\left(D^{r} V, D^{r} U_{x}\right)-\left(D^{r} V, D^{r} \hat{C}_{x} G(U)\right) \\
& \leqq\left\|D^{r} V\right\|_{0}^{2}+\frac{1}{2}\left\|D^{r} U_{x}\right\|_{0}^{2}+\frac{1}{2}\left\|D^{r} \partial_{x} G(U)\right\|_{0}^{2} .
\end{aligned}
$$


By the composition of functions inequality (cf. Kato, 1983, Lemma A3),

$$
\left\|D^{r} \partial_{x} G(U)\right\|_{0}^{2} \leqq\|G(U)\|_{r+1}^{2}=\left\|S_{\varepsilon} f(u)\right\|_{r+1}^{2} \leqq\|f(u)\|_{r+1}^{2} \leqq\left[\tilde{f}\left(\|u\|_{r}\right)\right]^{2}\|u\|_{r+1}^{2},
$$

where $\tilde{f}$ is a continuous, increasing function that depends only on $f$ and $r$. Using this in (2.15) gives the inequality

$$
\frac{1}{2} \frac{d}{d t}\left(\left\|D^{r} U_{x}\right\|_{0}^{2}+\left\|D^{r} V\right\|_{0}^{2}\right) \leqq \frac{1}{2}\left\|D^{r} U_{x}\right\|_{0}^{2}+\left\|D^{r} V\right\|_{0}^{2}+\left[\tilde{f}\left(\|u\|_{r}\right)\right]^{2}\|u\|_{r+1}^{2}
$$

Integrating (2.17) over the temporal interval $[0, t]$, letting $\varepsilon$ tend to zero, and using the dominated convergence theorem leads to an upper bound on $\left\|D^{r} \partial_{x} u\right\|_{0}^{2}+$ $\left\|D^{r} v\right\|_{0}^{2}$ of the form

$$
\begin{aligned}
& \left\|D^{r} \partial_{x} u(\cdot, t)\right\|_{0}^{2}+\left\|D^{r} v(\cdot, t)\right\|_{0}^{2} \leqq\left\|D^{r} \partial_{x} u_{0}\right\|_{0}^{2}+\left\|D^{r} v_{0}\right\|_{0}^{2} \\
& \quad+\int_{0}^{t}\left\{\left\|D^{r} \partial_{x} u(\cdot, \tau)\right\|_{0}^{2}+2\left\|D^{r} v(\cdot, \tau)\right\|_{0}^{2}+2\left(\tilde{f}\left(M_{r}\right)\right)^{2}\|u(\cdot, \tau)\|_{r+1}^{2}\right\} d \tau,
\end{aligned}
$$

where, for any real number $q, M_{q}$ is defined as

$$
M_{q}(T)=\sup _{0 \leqq t \leqq T}\left\{\|u(\cdot,) t\|_{q}+\|v(\cdot, t)\|_{q-1}\right\} .
$$

By hypothesis, $M_{1}(T) \leqq M_{0}$ for all $T<T^{*}$. By applying Gronwall's lemma to (2.18) and arguing inductively from the bound on $M_{1}(T)$, it is easily ascertained that $M_{r}(T)$ is uniformly bounded on $\left[0, T^{*}\right)$ for all $r$ such that $u_{0} \in H^{r}(\mathbb{R})$ and $v_{0} \in H^{r-1}(\mathbb{R})$. It follows that $\|u(\cdot, t)\|_{s}+\|v(\cdot, t)\|_{s-1}$ is uniformly bounded on $\left[0, T^{*}\right)$, and in consequence of our previous remarks, it must be the case that $T^{*}=+\infty$.

As mentioned at the outset, the proof for the system defined in (2.9) is in every respect similar.

In the next section, it is shown that the a priori estimate hypothesized in Theorem 4 can be provided for solutions corresponding to initial data that lies close enough to stable solitary waves.

\section{Nonlinear Stability of Solitary Waves}

In the special case of pure power nonlinearities, it is shown for a range of wave speeds that the solitary-wave solutions of (1.1) are stable in form. This means that a solution emanating from initial data near the solitary wave in question remains close to the orbit of that wave for as long as it exists.

As mentioned earlier, our proof of stability uses the Hamiltonian structure of the system (2.1), which we now explain. Let $(g, h)$ lie in $H^{s}(\mathbb{R}) \times H^{s-1}(\mathbb{R})$, where $s \geqq 1$. Define the Hamiltonian functional $H$ by

$$
H(g, h)=\int_{-\infty}^{\infty} \mathscr{H}(g(x), h(x)) d x,
$$

where the Hamiltonian density $\mathscr{H}$ is

$$
\mathscr{H}(g, h)=\frac{1}{2} h^{2}+\frac{1}{2} g^{2}+\frac{1}{2} g_{x}^{2}-F(g),
$$


and $F^{\prime}(z)=f(z)$ with $F(0)=0$. It is straightforward to check that the system (2.1) is formally equivalent to the Hamiltonian system

$$
\frac{d}{d t}\left(\begin{array}{l}
u \\
v
\end{array}\right)=\mathscr{J} \delta H(u, v)
$$

where $\mathscr{J}$ is the skew-adjoint operator

$$
\mathscr{J}=\left(\begin{array}{cc}
0 & \partial_{x} \\
\partial_{x} & 0
\end{array}\right)
$$

Here $\delta G$ denotes the gradient of the functional $G$ computed with respect to the $L^{2}(\mathbb{R})$-inner product. That is, if $G$ is a functional defined on $H^{1}(\mathbb{R}) \times L^{2}(\mathbb{R})$, then $\delta G$ has components $\delta_{u} G$ and $\delta_{v} G$ belonging to $H^{-1}(\mathbb{R}) \times L^{2}(\mathbb{R})$, which satisfy the relation

$$
\left.\frac{d}{d \varepsilon} G(u+\varepsilon \dot{u}, v+\varepsilon \dot{v})\right|_{\varepsilon=0}=\left\langle\dot{u}, \delta_{u} G\right\rangle_{1}+\left\langle\dot{v}, \delta_{v} G\right\rangle_{0}
$$

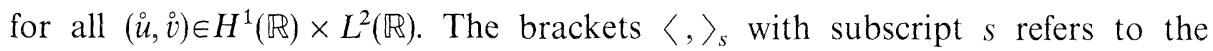
$H^{s}(\mathbb{R})-H^{-s}(\mathbb{R})$ dual pairing.

The system (2.1) is invariant under translation in $x$, so that if $(u(x, t), v(x, t))$ is a solution pair, then so is $\left(u\left(x+x_{0}, t\right), v\left(x+x_{0}, t\right)\right)$. This invariance corresponds to a commuting integral

$$
I(u, v)=\int_{-\infty}^{\infty} \mathscr{T}(u(x, t), v(x, t)) d x
$$

with density $\mathscr{T}(u, v)=u v$. Note that $H$ and $I$ are continuous, smooth functionals on $H^{1}(\mathbb{R}) \times L^{2}(\mathbb{R})$.

The solitary wave of speed $c$, if it exists, is a solution of (2.1) depending only on $\xi=x$-ct. Substituting $u(x, t)=U_{c}(x-c t)=U_{c}(\xi), v(x, t)=V_{c}(x-c t)=V_{c}(\xi)$ leads to the system

$$
\begin{aligned}
& -c U_{c}^{\prime}=V_{c}^{\prime}, \\
& -c V_{c}^{\prime}=\left(U_{c}-U_{c}^{\prime \prime}-f\left(U_{c}\right)\right)^{\prime},
\end{aligned}
$$

where ' connotes $\partial_{\xi}$. This system is recognized as having the variational form

$$
\mathscr{J} \delta(H+c I)\left(U_{c}, V_{c}\right)=0 .
$$

The characterization of the solitary wave $\left(U_{c}, V_{c}\right)$ of speed $c$ as a critical point of the functional $H+C I$ is crucial to the stability argument.

The proof of nonlinear stability follows from the abstract results of Grillakis, Shatah, and Strauss (1987). Particular results of this type may be found in Benjamin (1972), Bona (1975), Bennett et al. (1983), Weinstein (1986), Albert et al. (1987), and Bona et al. (1987). The basic idea, which goes back to Boussinesq (1877) is to use the invariant $(H+c I)(u, v)-(H+c I)\left(U_{c}, V_{c}\right)$ as a Lyapunov function to control the distance from $(u, v)$ to the orbit of $\left(U_{c}, V_{c}\right)$. Since $\delta(H+c I)=0$, when evaluated at the solitary wave, the second variation or Hessian determines the local behavior of the aforementioned invariant. For typical solitary-wave problems, this Hessian 
is never positive definite. Translational invariance mentioned earlier implies that zero will always be an eigenvalue of the Hessian with eigenfunction given by the $x$-derivative of the solitary wave (in the present case, $\left(U_{c}^{\prime}, V_{c}^{\prime}\right)$ ). Worse yet, solitary waves generally come in continuous families parametrized by the wave speed, and waves of different speeds will of course separate. In many cases, this corresponds to a negative eigenvalue. Alternatively, one may note that if $U_{\mathrm{c}}$ is even in $x$ (implying $U_{c}^{\prime}$ is odd) and the equation for the solitary wave reduces to a Sturm-Liouville problem, then the fact that $U_{c}^{\prime}$ has a zero implies that it is not the eigenfunction corresponding to the lowest eigenvalue. The potential difficulties associated with the lack of positivity of the Hessian are obviated by only asking for orbital stability, following Benjamin (1972). That is, provided that $(u(\cdot, 0), v(\cdot, 0))$ lies close to a given solitary wave, we shall only attempt to establish that $d\left((u, v),\left(U_{c}, V_{c}\right)\right)$ is small, for all $t$ for which $(u, v)$ exists, where

$$
d\left((u, v),\left(U_{c}, V_{c}\right)\right)=\inf _{y \in \mathbb{R}}\left\|(u(\cdot, t), v(\cdot, t))-\left(U_{c}(\cdot+y), V_{c}(\cdot+y)\right)\right\|_{H^{1}(\mathbb{R}) \times L^{2}(\mathbb{R})} \cdot
$$

(The choice of norm in (3.9) is dictated by the form of the Hessian and varies from problem to problem.) If (3.9) holds, the solitary wave is said to be stable in form in $H^{1}(\mathbb{R}) \times L^{2}(\mathbb{R})$.

Grillakis et al (1987, and see also Albert et al. 1987) prove a theorem which, when interpreted in the present context, yields the following result.

Theorem 5. Assume that (2.1) has a family of solitary waves which belong to $H^{3}(\mathbb{R}) \times H^{2}(\mathbb{R})$ as $c$ ranges between $c_{1}$ and $c_{2}$ with $c \rightarrow\left(U_{c}, V_{c}\right)$ a $C^{1}$ mapping of the interval $\left(c_{1}, c_{2}\right)$ into $H^{1}(\mathbb{R}) \times L^{2}(\mathbb{R})$. Moreover suppose that $U_{c}^{\prime}$ has one simple zero and decays rapidly to zero at $\pm \infty$. Then the solitary wave $\left(U_{c}, V_{c}\right)$ is stable in form in $H^{1}(\mathbb{R}) \times L^{2}(\mathbb{R})$ if the condition

$$
\frac{d^{2} m}{d c^{2}}(c)>0
$$

holds, where

$$
m(c)=(H+c I)\left(U_{c}, V_{c}\right) .
$$

Remarks. The quantity $m(c)$ is called the moment of instability, after a term used by Boussinesq in his discussion of solitary waves. Condition (3.10) is equivalent to the condition $d / d c I\left(U_{c}, V_{c}\right)>0$ since $\left(H^{\prime}+c I^{\prime}\right)\left(U_{c}, V_{c}\right)=0$.

Grillakis et al. also prove an abstract instability result, but technical difficulties associated with the operator $\mathscr{f}$ obstruct the direct application of their results to the case considered here.

The proof of Theorem 5 follows from the sufficiency part of Theorem 2 of Grillakis, Shatah, and Strauss. In the special case wherein translational symmetry is featured, their set-up includes a Hilbert space $\mathrm{X}$, a continuous Hamiltonian functional $H$, and a skew-adjoint, closed linear operator $\mathscr{J}$. Their results assert the validity of the stability criterion (3.10) if the following three assumptions hold.

Assumption 1. (Existence for the initial value problem) For each $U_{0} \in X$ there exists $t_{0}>0$, depending only on $\mu$, where $\left\|U_{0}\right\|_{X} \leqq \mu$, so that the Hamiltonian system 
$d U / d t=\mathscr{J} H^{\prime}(U)$ has a solution defined at least on $\left[0, t_{0}\right)$ such that $u(0)=u_{0}$, and for which $H(U(t))=H\left(U_{0}\right), I(U(t))=I\left(U_{0}\right)$ for $t \in\left[0, t_{0}\right)$.

Assumption 2. (Existence of solitary waves) There exist real numbers $c_{1}$ and $c_{2}$ with $c_{1}<c_{2}$ and a mapping $c \rightarrow \phi_{c}$ from the open interval $\left(c_{1}, c_{2}\right)$ into $X$ such that for each $c \in\left(c_{1}, c_{2}\right)$,

(i) $H^{\prime}\left(\phi_{c}\right)+c I^{\prime}\left(\phi_{c}\right)=0$,

(ii) $\phi_{c} \in D\left(\frac{d^{2}}{d x^{2}}\right)$ (that is, $\left.\phi_{c}^{\prime \prime} \in X\right)$, and

(iii) $\frac{d}{d x} \phi_{c} \neq 0$.

Assumption 3. (Spectrum of the Hessian) For each $c \in\left(c_{1}, c_{2}\right), L_{c}=\left(H^{\prime \prime}+c I^{\prime \prime}\right)\left(U_{c}, V_{c}\right)$ has exactly one negative eigenvalue which is simple, has its kernel spanned by $d_{x} \phi_{c}$ and the rest of its spectrum is bounded away from zero.

Thus Theorem 5 will hold if these assumptions hold. For the case under consideration here, Assumption 1 holds in a slightly modified form as proved in the previous section. Namely, it was shown to hold in the dense subspace $H^{s+2}(\mathbb{R}) \times H^{s+1}(\mathbb{R})$ for some $s>1 / 2$ rather than $H^{1}(\mathbb{R}) \times L^{2}(\mathbb{R})$. This does not alter the stability proof if the initial data is restricted to lie in this subspace and the evolution takes place there as is the case for our examples. The existence of solitary waves is assumed in Theorem 5 so that Assumption 2 holds.

To verify Assumption 3, compute the Hessian operator $L_{c}$ by calculating the associated quadratic form, which is denoted by $Q_{c}$. By definition, $Q_{c}(g, h)$ is the coefficient of $\varepsilon^{2}$ in $H\left(U_{c}+\varepsilon g, V_{c}+\varepsilon h\right)+c I\left(U_{c}+\varepsilon g, V_{c}+\varepsilon h\right)$, and so is given by

$$
\begin{aligned}
& \int_{-\infty}^{\infty}\left\{\frac{1}{2}\left(g^{2}+\left(g_{x}\right)^{2}+h^{2}\right)-\frac{1}{2} f^{\prime}\left(U_{c}\right) g^{2}+c g h\right\} d x \\
& =\int_{-\infty}^{\infty}\left\{\frac{1}{2}\left(\left(1-c^{2}\right) g^{2}+\left(g^{\prime}\right)^{2}-f^{\prime}\left(U_{c}\right) g^{2}\right)+\frac{1}{2}(h+c g)^{2}\right\} d x \\
& \equiv Q_{c}^{(1)}(g)+\frac{1}{2}\|h+c g\|_{0}^{2} .
\end{aligned}
$$

Note that $Q_{c}$ is therefore the sum of a Sturm-Liouville form $Q_{c}^{(1)}$ and the non-negative term $\frac{1}{2}\|h+c g\|_{0}^{2}$. From Eqs. (3.7) for the solitary wave $\left(U_{c}, V_{c}\right)$ it follows that $g=U_{c}^{\prime}, h=V_{c}^{\prime}$ satisfies $L_{c}(g, h)=0$. To see that it is the only eigenfunction with eigenvalue 0 , view the quadratic form $Q_{c}(g, h)$ as the pairing of $(g, h)$ against $(\tilde{g}, \tilde{h})$ in the $H^{1}(\mathbb{R}) \times L^{2}(\mathbb{R})-H^{-1}(\mathbb{R}) \times L^{2}(\mathbb{R})$ duality, where $(\tilde{g}, \tilde{h})^{t}$ is the unbounded operator

$$
\tilde{L}=\left[\begin{array}{ll}
\frac{1}{2}\left(1-\partial_{x}^{2}-f^{\prime}\left(U_{c}\right)\right) & \frac{c}{2} \\
\frac{c}{2} & \frac{1}{2}
\end{array}\right]
$$

applied to $(g, h)^{t}$. Then $\tilde{L}(g, h)^{t}=0$ implies that $h=-c g$ and $\frac{1}{2}\left(1-c^{2}-\partial_{x}^{2}-f^{\prime}\left(U_{c}\right)\right) g=0$. The second solution to this ordinary differential equation grows exponentially 
since $U_{c}^{\prime}$ decays exponentially, hence the kernel of $L_{c}$ in $H^{1}(\mathbb{R}) \times L^{2}(\mathbb{R})$ is spanned by $\left(U_{c}^{\prime}, V_{c}^{\prime}\right)$.

To prove that there is a single, simple, negative eigenvalue, consider $Q_{c}^{(1)}$ defined in (3.11) above. By Sturm-Liouville theory, since $U_{c}^{\prime}$ has a single, simple zero by hypothesis, there is precisely one eigenfunction corresponding to an eigenvalue smaller than zero, say $G_{1}$. Since $Q_{c}^{(1)}$ achieves a negative value, so does $Q_{c}$ and therefore the lowest eigenvalue of $L_{c}$ is negative. If $\lambda_{1}$ is the lowest eigenvalue of $L_{c}$, Assumption 3 will hold if it is shown that the next eigenvalue $\lambda_{2}$ is 0 , which is known to be simple, and it is shown that $\lambda_{3}$ is in fact strictly positive. These results are proved using the min-max characterization of eigenvalues (Courant and Hilbert, 1953, Chap. VI), namely

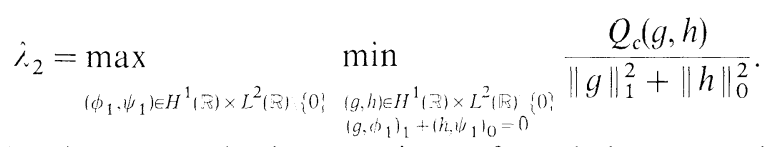

Choose $\phi_{1}=G_{1}, \psi_{1}=0$ to obtain an estimate from below, namely

$$
\lambda_{2} \geqq \min _{\substack{(g h) \in H^{1}(B) \times L^{2}(\&)\{0\} \\\left(g, G_{1} 1_{1}=0\right.}} \frac{Q_{c}(g, h)}{\|g\|_{1}^{2}+\|h\|_{0}^{2}} .
$$

The right-hand side of (3.14) is non-negative since on the displayed subspace, $Q_{c}^{(1)}(g) \geqq 0$ by Sturm-Liouville theory. Thus $\lambda_{2}=0$ and, from earlier considerations, $\lambda_{2}$ is simple. To estimate $\lambda_{3}$ from below by a strictly positive quantity, the same argument is used with respect to the two dimensional subspace spanned by $\left(G_{1}, 0\right)$, $\left(U_{c}^{\prime}, 0\right)$. This completes the proof of Theorem 5 .

To obtain useful information from Theorem 5 one must demonstrate the existence of solitary waves and compute the moment of instability

$$
m(c) \equiv(H+c I)\left(U_{c}, V_{c}\right)
$$

explicitly (or at least prove $m^{\prime \prime}(c)>0$ ). In general, this may be non-trivial. For the special case of pure power nonlinearities, complete answers may be obtained quite readily.

By a pure power nonlinearity, we mean that $f(u)=u^{p}$ for some integer $p$. In this case, the solitary-wave solution of (2.1a) is given explicitly by

$$
U_{c}(\xi)=A \operatorname{sech}^{2 /(p-1)}(B \xi), \quad V_{c}(\xi)=-c A \operatorname{sech}^{2 /(p-1)}(B \xi),
$$

where the positive roots are meant in both cases,

$$
\begin{gathered}
A=A(c, p)=\left[\left(\frac{p+1}{2}\right)\left(1-c^{2}\right)\right]^{1 /(p-1)}, \\
B=B(c, p)=\frac{\left(1-c^{2}\right)^{1 / 2}(p-1)}{2},
\end{gathered}
$$

and $\xi=x-c t$ as before. For this case, Theorem 5 has the following corollary.

Theorem 6. For $p>1$, the system

$$
\begin{aligned}
& u_{t}=v_{x}, \\
& v_{t}=\left(u-u_{x x}-u^{p}\right)_{\imath}
\end{aligned}
$$


has solitary waves $\left(U_{c}, V_{c}\right)$ given by formulas (3.16) for all speeds $c$ such that $c^{2}<1$. For $p<5$, these solutions are stable in the $H^{1}(\mathbb{R}) \times L^{2}(\mathbb{R})$ norm for speeds $c$ such that $(p-1) / 4<c^{2}<1$. In particular, when $p=2$ (the classical case), the solitary wave is stable for speeds $c$ such that $1 / 4<c^{2}<1$.

Remarks. For general, smooth functions $f(u)$, the stability criterion may be computed at least implicitly by eliminating $V_{c}$ and expressing $U_{c}$ as an implicit integral using the relation

$$
\left(1-c^{2}\right) \frac{U_{c}^{2}}{2}-\frac{\left(U_{c}^{\prime}\right)^{2}}{2}-F\left(U_{c}\right)=0
$$

For $f(u)$ sufficiently close to a pure power, one may also use continuity to decide the stability of the solitary wave.

Proof. The hypotheses of Theorem 5 are verified easily from the explicit form (3.16) of the solitary wave. The smoothness in $c$ of the family $\left(U_{c}, V_{c}\right)$ is immediate as is the rapid decay and the fact that $U_{c}^{\prime}$ has a simple zero.

Applying the stability criterion gives the condition $(p-1) / 4<c^{2}<1$. This follows by computing explicitly

$$
\begin{aligned}
m(c) & =(H+c I)\left(U_{c}, V_{c}\right)=\int_{-\infty}^{\infty}\left\{\frac{1}{2}\left(U_{c}^{2}+\left(U_{c}^{\prime}\right)^{2}+V_{c}^{2}\right)-\frac{U_{c}^{p+1}}{p+1}+c U_{c} V_{c}\right\} d \xi \\
& =\int_{-\infty}^{\infty}\left(U_{c}^{\prime}\right)^{2} d \xi=\frac{4}{(p-1)^{2}} A^{2} B \int_{-\infty}^{\infty} \operatorname{sech}^{4 /(p-1)}(y) \tanh ^{2}(y) d y \\
& =K_{p}\left(1-c^{2}\right)^{(p+3) / 2(p-1)}
\end{aligned}
$$

for some positive constant $K_{p}$. Here the system (3.7) was used to eliminate $V_{c}$ and (3.18) was used to simplify the resulting expression in the second line. It is then simple to check that the second derivative of $\left(1-c^{2}\right)^{(p+3) / 2(p-1)}$ is positive exactly when $c^{2}>(p-1) / 4$.

Remark. It is worth noting that the stability theory presented above goes over intact for non-integer values of $p$ in the range $1<p<5$, provided that we define $f(u)=|u|^{p-1} u$. Indeed, with only the occasional insertion of absolute values, the proof proceeds line for line as given above.

While this latter point appears to have little practical interest since nonlinearities arising in physical and mechanical models feature integer powers of the dependent variable, it is interesting enough mathematically to warrant a formal statement which we designate a corollary to the proof of Theorem 5.

Corollary 7. For any real number $p>1$, the system

$$
\begin{aligned}
& u_{t}=v_{\lambda}, \\
& v_{t}=\left(u-u_{x x}-|u|^{p-1} u\right)_{\lambda},
\end{aligned}
$$

has solitary-wave solutions $\left(U_{c}, V_{c}\right)$ given by formulas (3.16) for all speeds c with $c^{2}<1$. These solutions are stable in the $H^{1}(\mathbb{R}) \times L^{2}(\mathbb{R})$-norm for all speeds $c$ with $(p-1) / 4<c^{2}<1$. 


\section{A Global Existence Theorem}

In this section it is shown that if $p<5$, and the initial data $\left(u_{0}, v_{0}\right)$ lies close enough to the initial data $\left(U_{c}, V_{c}\right)$ corresponding to a stable solitary wave, then the local solution of (2.1), guaranteed by Theorem 1 admits a unique extension to a global smooth solution. As far as we are aware, this is the only global well-posedness theorem pertaining to this type of nonlinear string equation.

Here is a precise statement of the result in view.

Theorem 8. Let $s \geqq 1, p<5$, and $(p-1) / 4<c^{2}<1$. Let $\left(U_{c}, V_{c}\right)$ denote a solitarywave solution of $(2.1)$ or, if $p$ is not an integer, of (3.20) corresponding to these values of $p$ and $c$. Then there exists $\delta=\delta(p, c)>0$ such that it $\left(u_{0}, v_{0}\right) \in H^{s+2}(\mathbb{R}) \times H^{s+1}(\mathbb{R})$, and there is a number $\theta$ such that

$$
\left\|u_{0}(\cdot)-U_{c}(\cdot+\theta)\right\|_{1}+\left\|v_{0}(\cdot)-V_{c}(\cdot+\theta)\right\|_{0} \leqq \delta,
$$

then the solution $(u, v)$ of $(2.1)$ or (3.20) corresponding to the initial data $\left(u_{0}, v_{0}\right)$ is global and lies in $X_{s}(T) \times X_{s-1}(T)$ for all positive $T$. Moreover, for all $T>0$, the mapping sending $\left(u_{0}, v_{0}\right)$ to the solution $(u, v)$ of $(2.1)$ or $(3.20)$ is continuous from $H^{s+2}(\mathbb{R}) \times H^{s+1}(\mathbb{R})$ into $X_{s}(T) \times X_{s-1}(T)$.

Proof. Let $T^{*}$ be the maximal time of existence of the solution $(u, v)$. The goal is to show that $T^{*}=\infty$, which means that the solution exists for all $t>0$. By Theorem 4 , it suffices to show that the pair $(u, v)$ remains bounded in $H^{1}(\mathbb{R}) \times L^{2}(\mathbb{R})$ for all $0 \leqq t \leqq T<T^{*}$ with bound independent of $T$. This is true for all initial values sufficiently close to a stable solitary wave by Theorem 6 . Thus the proof is finished.

In terms of the scalar equation (1.1) this yields

Corollary 9. Let $s \geqq 1, p<5$, and $(p-1) / 4<c^{2}<1$. Let $U_{c}$ denote the solitary-wave solution of (1.1) with $f(u)$ either $u^{p}$, p integer, or $|u|^{p-1} u$ for $p$ real. Then there exists $a \delta=\delta(p, c)>0$ such that if $\left(u_{0}, u_{1}\right) \in H^{s+2}(\mathbb{R}) \times H^{s}(\mathbb{R}), u_{1}=v_{x}$ for some $v \in H^{s+1}(\mathbb{R})$, and there is a number $\theta$ such that

$$
\left\|u_{0}(\cdot)-U_{c}(\cdot+\theta)\right\|_{1}+\left\|v(\cdot)+c U_{c}(\cdot+\theta)\right\|_{0}<\delta
$$

then the solution of (1.1) corresponding to the initial data $\left(u_{0}, u_{1}\right)$ is global and lies in $Y_{s}(T)$ for all positive $T$. Moreover, for all $T>0$, the mapping sending $\left(u_{0}, u_{1}\right)$ to the solution $u$ of $(1.1)$ is continuous from the relevant subspace of $H^{s+2}(\mathbb{R}) \times H^{s}(\mathbb{R})$ into $Y_{s}(T)$.

\section{Conclusion}

A local existence theory and a stability theory for solitary waves has been established for the nonlinear, dispersive string equation (1.1), and for its system analog (2.1). In the special case wherein the nonlinearity is a pure power, the stability theory is known to apply, and to imply the existence of global solutions emanating from data that is close to a stable solitary wave.

The global existence theory is interesting for at least two reasons. First, solutions of the same equations without the dispersive term (the term $-u_{x x x x}$ in 1.1 a) typically lose regularity in finite time, a fact that becomes clear upon consideration of the 
characteristics associated with the non-dispersive equation. Hence, as with other nonlinear evolution equations, dispersion is seen to counteract the steepening effects of unbridled nonlinearity.

However, unlike some nonlinear, dispersive evolution equations, global existence does not subsist just upon smoothness and decay properties of the initial data. Thus our theory is especially interesting when considered in juxtaposition with the blow-up results of Kalantarov and Ladyzhenskaya (1978). These authors prove that certain solutions of (1.1) (and many other equations) cannot exist for all time, thus suggesting strongly that something other than straightforward energy-type estimates are needed to establish global existence. The non-global solutions of Kalantarov and Ladyzhenskaya occur for negative-energy initial data which satisfies an additional hypothesis. Our results indicate that something like their hypotheses are needed to deduce blow-up.

For the special case $p=2$, the methods of inverse scattering apply (Zakharov, 1974; Deift et al. 1982). Certain global solutions are then found, including multi-solitons and a special class of complex-valued solutions (Deift et al 1982). However, a theory of well-posedness centered around these ideas appears to be difficult, though the formal inference that the evolution of initial data is carried in part by a finite number of solitary waves is certainly intriguing. On the basis of their blow-up results, Kalantarov and Ladyzhenskaya (1978) called into question the efficacy of the inverse-scattering theory as applied to the nonlinear string equation. The discovery reported here that some of the solitary-wave solutions are stable would seem to indicate that if smooth initial data is predicted by the inverse-scattering theory to resolve itself into solitary waves in the stable range, then the resulting solution will probably be global and smooth, so that the calculations made using the inverse-scattering theory will apply to it for all time.

Acknowledgement. The authors want to thank P. Deift for helpful discussions and for simplifying the argument in the proof of Theorem 5 .

\section{References}

Albert, J., Bona, J. L., Henry, D.: Sufficient conditions for stability of solitary-wave solutions of model equations for long waves. Physica 24D, 343-366 (1987)

Benjamin, T. B.: The stability of solitary waves. Proc. Roy. Soc. Lond. A328, 153-183 (1972)

Bennett, D. P., Bona, J. L., Brown, S. E., Stansfield, D. D., Stroughair. J. D.: The stability of internal solitary waves. Math. Proc. Camb. Phil. Soc. 94, 351- 379 (1983)

Berryman, J.: Stability of solitary waves in shallow water. Phys. Fluids 19, 771-777 (1976)

Bona, J. L.: On the stability theory of solitary waves. Proc. Roy. Soc. Lond. A344, 363-374 (1975)

Bona, J. L.. Smith, R.: A model for the two-way propagation of water waves in a channel. Math. Proc. Camb. Phil. Soc. 79, 167-182 (1976)

Bona, J. L., Souganidis, P., Strauss, W.: Stability and instability of solitary waves of KdV type. Proc. Roy. Soc. Lond. A411, 395-412 (1987)

Boussinesq, J.: Théorie des ondes et de remous qui se propagent....J. Math. Pures Appl., Sect. 2, 17, 55-108 (1872)

Boussinesq, J.: Essai sur la théorie des eaux courantes. Mem. prés. div. Sav. Acad. Sci. Inst. Fr. 23, $1-680(1877)$

Courant, R., Hilbert, D.: Methods of mathematical physics Vol. 1. New York: Interscience 1953 
Deift, P., Tomei, C., Trubowitz, E.: Inverse scattering and the Boussinesq equation. Commun. Pure Appl. Math. 35, 567-628 (1982)

Grillakis, J., Shatah, J., Strauss, W. A.: Stability theory of solitary waves in the presence of symmetry I. J. Funct. Anal. 74, 160-197 (1987).

Kalantarov, V. K., Ladyzhenskaya, O. A. The occurrence of collapse for quasilinear equations of parabolic and hyperbolic types. J. Sov. Math. 10, 53-70 (1978)

Kato, T.: Quasilinear equations of evolution, with applications to partial differential equations. Lecture Notes in Mathematics Vol. 448, pp. 25-70. Berlin, Heidelberg, New York: Springer 1974

Kato, T.: On the Cauchy problem for the (generalized) Korteweg-de-Vries equation. Studies in Applied Mathematics. Ad. Math. Suppl. Stud. 8, 93-128 (1983)

Lions, J.-L., Magenes, E.: Problèmes aux limites non homogènes et applicatıons, Vol. I. Parıs: Dunod 1968

Weinstein, M.: Lyapunov stability of ground states of nonlinear dispersive evolution equations. Commun. Pure Appl. Math. 39, 51-68 (1986)

Zakharov, V. E.: On the stochastization of one-dimensional chains of nonlinear oscillators. Sov. Phys. JETP 38, 108-110 (1974)

Communicated by C. H. Taubes

Received July 31, 1987; in revised form January 30, 1988 
